\title{
FITOSSANIDADE
}

\section{RESISTÊNCIA DE PLANTAS DE COFFEA ARABICA, COFFEA CANEPHORA E HÍBRIDOS INTERESPECÍFICOS À CERCOSPORIOSE (1)}

\author{
FLÁVIA RODRIGUES ALVES PATRICIO (2); MASAKO TOMA BRAGHINI ( $\left.{ }^{3}\right)$; \\ LUIZ CARLOS FAZUOLI ( $\left.{ }^{3}\right)$
}

\begin{abstract}
RESUMO
O presente estudo objetivou avaliar a resistência de plantas de Coffea arabica, C. canephora e de híbridos interespecíficos, à cercosporiose (Cercospora coffeicola), importante doença da cultura do cafeeiro. Foram realizados dois experimentos em 2003, em Campinas (SP), com mudas das cultivares de C. arabica, Mundo Novo IAC 388-17-1, Mundo Novo IAC 376-4, Bourbon Amarelo, Bourbon Vermelho, Obatã IAC 1669-20, Catuaí Vermelho IAC 144, Catuaí Amarelo IAC 62, Icatu Precoce IAC 3282, Icatu Vermelho IAC 4045, Ouro Verde IAC H5010-5 e Tupi IAC 1669-33; de C. canephora, Robusta IAC 1653-7 e Apoatã IAC 2258, além de dois híbridos interespecíficos Piatã IAC 387 e Híbrido de Timor IAC 1559-13. As mudas foram submetidas à inoculação com uma suspensão de conídios preparada com cinco isolados coletados em diferentes regiões do Estado de São Paulo. Os materiais mais suscetíveis foram Robusta IAC 1653-7, A poatã IAC 2258, Híbrido de Timor IAC 1559-13, Bourbon Vermelho e Bourbon Amarelo com incidência entre $63,1 \%$ e $81,6 \%$ no primeiro experimento e entre $70 \%$ e 93,3\% no segundo e severidade (notas 1-6) entre 2,92 e 3,42 no primeiro experimento e entre 3,64 e 2,79 no segundo. Os materiais mais resistentes foram Piatã IAC 387, Ouro Verde IAC H5010-5 e Tupi IAC 1669-33, com incidência entre 28,4\% e 35,5\% no primeiro experimento e $21,1 \%$ e 55,6\% no segundo, e severidade entre 1,44 e 1,73 e entre 1,28 e 1,88, respectivamente, no primeiro e segundo experimentos.
\end{abstract}

Palavras-chave: Cercospora coffeicola, Coffea arabica, resistência a doenças.

\section{ABSTRACT \\ RESISTANCE OF PLANTS OF COFFEA ARABICA, COFFEA CANEPHORA AND INTERESPECIFIC HYBRIDS TO BROWN EYE SPOT}

In the present study plants of Coffea arabica, C. canephora, and interespecific hybrids were evaluated on their response to brown eye spot (Cercospora coffeicola), an important disease of the coffee crop. Two experiments were carried out in 2003 in Campinas, SP, with seedlings of C. arabica cultivars Mundo Novo IAC 388-17-1, Mundo Novo IAC 376-4, Bourbon Amarelo, Bourbon Vermelho, Obatã IAC 1669-20, Catuaí Vermelho IAC 144, Catuaí Amarelo IAC 62, Icatu Precoce IAC 3282, Icatu Vermelho IAC 4045, Ouro Verde IAC H5010-5, Tupi IAC 1669-33, of C. canephora, Robusta IAC 1653-7 and Apoatã IAC 2258, as well as two interspecific hybrids Piatã IAC 387 and Híbrido de Timor IAC 1559-13. The seedlings were inoculated with a conidial suspension containing a mixture of five isolates of $C$. coffeicola collected in different regions of São Paulo State. The most susceptible materials were Robusta IAC 1653-7, Apoatã IAC 2258, Híbrido de Timor IAC 1559-13, Bourbon Vermelho, and Bourbon Amarelo, with levels of incidence that varied from 81.6 to $63.1 \%$ in the first experiment, and from 70 to $93.3 \%$ in the second, and severity (graded scale 1-6) from 2.92 and 3.42 in the first experiment to 2.79 and 3.64 in the second. The most resistant materials were Piatã IAC 387, Ouro Verde IAC H5010-5 and Tupi IAC 1669-33, with incidence between 28.4 and $35.5 \%$ in the first experiment and between 21.1 and $55.6 \%$, in the second, and severity that varied from 1.44 to 1.73 in the first experiment to 1.28 to 1.88 in the second.

Key words: Cercospora coffeicola, Coffea arabica, disease resistance.

(1) Recebido para publicação em 24 de abril de 2009 e aceito em 15 de março de 2010.

( ${ }^{2}$ Instituto Biológico, Caixa Postal 70, 13012-970 Campinas (SP). Email: flavia@biologico.sp.gov.br (*) Autora correspondente.

( ${ }^{3}$ Centro de Análise e Pesquisa Tecnológica do Agronegócio do Café "Alcides Carvalho". Instituto Agronômico, Caixa Postal 28, 13001-970 Campinas (SP). E-mail: mako@iac.sp.gov.br; fazuoli@iac.sp.gov.br 


\section{INTRODUÇÃO}

A cercosporiose, também conhecida como mancha-de-olho-pardo, causada por Cercospora coffeicola Berk. \& Cooke, é uma doença endêmica em todas as regiões produtoras de café do Brasil (ZAMBolim et al., 2005). Atualmente, está entre as principais doenças da cultura no cerrado (CARVAlHo et al., 2002) e sua importância tem aumentado nas demais regiões cafeicultoras (ThomAZIello et al., 2000). Os sintomas da doença manifestam-se na forma de lesões nas folhas e nos frutos. As folhas afetadas caem rapidamente e as lesões nos frutos prejudicam a qualidade da bebida (ZамвоLIM et al., 2005). Em viveiros, a desfolha pode ser intensa e atrasar o desenvolvimento das mudas (ZAмвоLim et al., 2005). Cafezais implantados em solos arenosos, ou com nutrição deficiente ou desequilibrada, especialmente na relação $\mathrm{N} / \mathrm{K}$, são mais predispostos à cercosporiose (ZAMBOLIM et al., 2005; CARVALHO et al., 2002).

O controle da cercosporiose é realizado desde a formação das mudas, de forma preventiva, mediante a utilização de substratos adubados adequadamente. Nos viveiros, muitas vezes, o controle químico é necessário, especialmente antes da aclimatação das mudas para serem levadas ao campo (ZAmBolim et al., 2005). Em condições de campo, recomenda-se evitar solos arenosos e manter rigoroso controle das adubações durante o ciclo da cultura (ZAMBolim et al., 2005), mas a utilização de fungicidas para o controle da cercosporiose é, atualmente, parte integrante dos programas de tratamento fitossanitário do cafeeiro (ThomAZiello et al., 2000).

Importantemedidadecontroleparaacercosporiose e outras doenças do cafeeiro é a utilização de cultivares resistentes. Entretanto, a resistência à cercosporiose está sendo pouco estudada no Brasil, provavelmente porque os esforços da pesquisa se concentraram no desenvolvimento de cultivares resistentes à ferrugem, a mais importante doença da cultura, ou pelo fato de ser essa doença considerada de pouca importância para a cultura ou estava relacionada com deficiências na nutrição dos cafeeiros. FERNANDEs et al. (1990) verificaram que, dentre 27 progênies de Catimor, cruzamento de Híbrido de Timor e Caturra, cinco progênies, UFV 2870,UFV 2875, UFV 2876, UFV 3876 e UFV 4180, bem como a cultivar Catuaí Vermelho IAC 144, utilizada como testemunha, tiveram menor severidade da doença, embora nenhum dos genótipos avaliados tenha sido completamente resistente à cercosporiose. PozzA et al. (2004) observaram que mudas de cafeeiro da cultivar Icatu submetidas à inoculação com C. coffeicola foram menos afetadas pela cercosporiose do que mudas das cultivares Catuaí e Mundo Novo, que não diferiram entre si.
Considerando que a cercosporiose é uma doença do cafeeiro cuja importância tem aumentado, especialmente nas regiões de expansão da cafeicultura como o cerrado e na cafeicultura irrigada, a identificação de fontes de resistência a esta doença se torna cada vez mais relevante. Diante do exposto, este trabalho objetivou avaliar a resistência à cercosporiose de cultivares de cafeeiro utilizadas em grande escala na cafeicultura brasileira, bem como de materiais empregados nos programas de melhoramento como fonte de resistência à ferrugem, ao bicho mineiro e a nematóides do gênero Meloidogyne spp.

\section{MATERIAL E MÉTODOS}

Dois experimentos foram desenvolvidos em casa de vegetação e viveiro em Campinas (SP), com mudas de 11 cultivares de cafeeiros pertencentes à espécie Coffea arabica, duas seleções de C. canephora, além de dois híbridos interespecíficos (Tabela 1). O primeiro experimento foi realizado de abril a maio e o segundo, de junho a agosto de 2003.

Mudas com seis meses de idade foram utilizadas no primeiro experimento e com oito meses no segundo. As mudas foram preparadas simultaneamente em condições protegidas de casa de vegetação, com irrigação manual e isoladas de outras plantas de café. Os recipientes plásticos usados para a produção das mudas continham um litro de substrato preparado de acordo com as recomendações técnicas (THOMAZIELlo et al.,2000). Não foi realizada a aplicação de pesticidas antes e durante a realização dos experimentos.

Para a inoculação das mudas, em ambos os experimentos, foi utilizada uma suspensão de conídios composta pela mistura, em partes iguais, de cinco isolados de C. coffeicola, obtidos em diferentes regiões do Estado de São Paulo. Os isolados utilizados foram: IB 01/02 (coletado de plântulas de cafeeiro de um viveiro de Itapira, SP); IB02/ 02 (coletado em mudas de cafeeiro produzidas no Instituto Biológico em Campinas, SP), IB03/ 02 (coletado em plântulas de um viveiro de Garça, $\mathrm{SP}) ; \mathrm{IB} 04 / 02$ (coletado em plântulas do viveiro do IAC, em Campinas, SP) e IB05/02 (coletado em lavoura com cinco anos de idade no município de Presidente Prudente, SP).

O fungo foi cultivado em meio de cultura V-8 (DHINGRA e Sinclair, 1995) por cinco dias em ambiente de laboratório, sob luz fluorescente contínua. As suspensões utilizadas para as inoculações foram preparadas raspando-se com um pincel de cerda fina a superfície do meio de cultura, após a colocação de aproximadamente $10 \mathrm{~mL}$ de água em cada placa para retirar os conídios. Após a filtragem em peneira de cozinha, sobreposta com uma camada de gaze, foi realizada a contagem de 
Tabela 1. Plantas de Coffea arabica, C. canephora e híbridos interespecíficos testados para resistência à cercosporiose

\begin{tabular}{|c|c|c|}
\hline Espécies e híbridos interespecíficos & Cultivares & Citação \\
\hline Coffea arabica (tetraploide $2 \mathrm{n}=4 \mathrm{x}=44$ cromossomos) & Bourbon Amarelo & FAZUOLI (1999) \\
\hline C. arabica & Bourbon Vermelho & GuERREIRO FilHo et al. (2006) \\
\hline C. arabica & Mundo Novo IAC 388-17-1 & FAZUOLI (1999) \\
\hline C. arabica & Mundo Novo IAC 376-4 & FAZUOLI (1999) \\
\hline C. arabica & Catuaí Vermelho IAC 144 & FAZUOLI (1999) \\
\hline C. arabica & Catuaí Amarelo IAC 62 & FAZUOLI (1999) \\
\hline C. arabica & Icatu Precoce IAC 3282 & FAZUOLI (1999) \\
\hline C. arabica & Icatu Vermelho IAC 4045 & FAZUOLI (1999) \\
\hline C. arabica & Obatã IAC 1669-20 & FAZUOLI (1999) \\
\hline C. arabica & Ouro Verde IAC H5010-5 & Aguiar et al. (2004) \\
\hline C. arabica & Tupi IAC 1669-33 & Aguiar et al. (2004) \\
\hline C. canephora $(2 \mathrm{n}=2 \mathrm{x}=22$ cromossomos $)$ & Apoatã & FAZUOLI et al. (2008). \\
\hline C. canephora & Robusta IAC1653-7 & GuerReIro FilHo et al. (2006). \\
\hline C. arabica $\mathrm{x}$ C. canephora $(2 \mathrm{n}=4 \mathrm{x}-\mathrm{=}=44$ cromossomos $)$ & Hibrido de Timor IAC 1559-13 & Silvarolla et al. (1997) \\
\hline C. arabica $\times$ C. dewevrei & Piatã IAC 387 & MEdina FilHo et al. (1995) \\
\hline
\end{tabular}

conídios em câmara de Neubauer e as suspensões foram diluídas à concentração de $10^{4}$ conídios de C. coffeicola $\mathrm{mL}^{-1}$, sendo então aspergidas sobre as faces superior e inferior das folhas.

No primeiro experimento, todas as folhas das mudas foram submetidas à inoculação. No segundo, apenas os três últimos pares de folhas das plântulas foram infectados. Logo após a inoculação, as mudas foram mantidas sob câmara úmida por 72 horas, em casa de vegetação com temperatura de $25 \pm 2{ }^{\circ} \mathrm{C}$. Após a retirada da câmara úmida, as mudas permaneceram na casa de vegetação por mais dois dias sob regime de microaspersão e com a umidade relativa do ar em torno de $80 \%$, sendo então transferidas para viveiro, no qual também foram mantidas sob regime de microaspersão.

Os experimentos foram realizados em delineamento inteiramente casualizado, com 20 repetições para o primeiro experimento e 15 para o segundo, em função da disponibilidade de mudas. Cada repetição foi representada por uma muda.

No primeiro experimento, as plantas foram avaliadas 17 dias após a inoculação, sendo todas as folhas examinadas. No segundo experimento, foram realizadas duas avaliações aos 10 e 16 dias após a inoculação. Foram avaliadas a incidência e a severidade da cercosporiose, sendo a severidade avaliada por uma escala de notas de 1 a 6, desenvolvida pelos avaliadores no momento da primeira avaliação do primeiro experimento (Figura 1). As folhas representativas de cada nível da escala foram desenhadas em filme transparente. A porcentagem de área afetada para cada nível da escala foi estimada por meio da leitura da área das folhas e da área ocupada pelas lesões em aparelho LICor modelo LI-3050A/4.
Os dados foram analisados pela nota média da doença calculada segundo a fórmula: nota média $=\left(n_{1} \times 1+n_{2}\right.$ $\left.\times 2+n_{3} \times 3+n_{4} \times 4+n_{5} \times 5+n_{6} \times 6\right) / N$ em que $n_{1^{\prime}} n_{2^{\prime}}$ $\mathrm{n}_{3,}, \mathrm{n}_{4,} \mathrm{n}_{5}, \mathrm{n}_{6}=$ número de folhas da planta com as notas $1,2,3,4,5$ e 6 ; e $\mathrm{N}=$ número total de folhas da amostra (MORAES, 2008).

Os dados foram submetidos à análise de variância, e as médias comparadas pelo teste de Scott-Knott a 5\% de probabilidade. Os dados relativos à incidência do primeiro experimento e da primeira e segunda avaliações do segundo experimento foram transformados para $\sqrt{x}+1$, antes da realização das análises.

\section{RESULTADOS E DISCUSSÃO}

No primeiro experimento, observou-se menor incidência da cercosporiose nos cafeeiros das cultivares Tupi IAC 1669-33, Ouro Verde IAC H5010-5, Catuaí Vermelho IAC 144 e Piatã IAC 387, respectivamente com 28,4; 31,2; 33,8 e 35,5\% de incidência. (Tabela 2). A progênie Robusta IAC1653-7 (C. canephora) e as cultivares Apoatã IAC 2258, Bourbon Vermelho e Bourbon Amarelo tiveram os maiores valores de incidência da doença, com 81,6;74,8; 70,0 e 63,1\% respectivamente. Com relação à severidade, os menores valores foram observados nas cultivares Tupi IAC 1669-33 (1,47), Ouro Verde IAC H5010-5 (1,65), Piatã IAC 387 (1,73) e Catuaí Vermelho IAC 144 (1,75), sendo seguidos por Icatu Vermelho IAC $4045(2,07)$ e Obatã IAC 1669-20 (2,27). A progênie Robusta IAC1653-7 (C. canephora) e as cultivares Bourbon Vermelho e Bourbon Amarelo tiveram os valores mais elevados de severidade da doença, com notas de 3,42; 3,37 e 2,92 respectivamente (Tabela 2 ). 

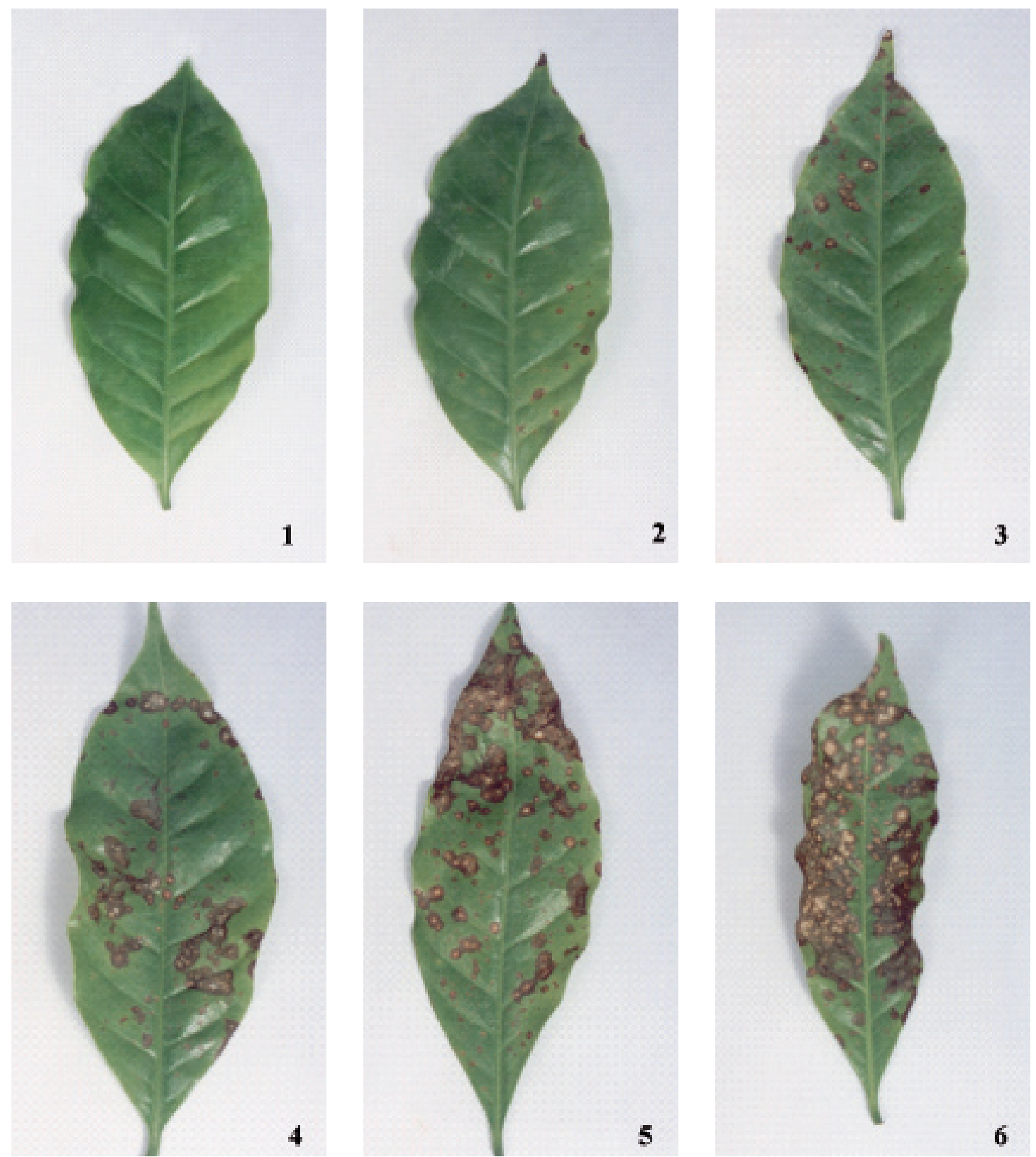

Figura 1. Escala de notas de 1 a 6 utilizada para avaliar a severidade da cercosporiose. As notas representam diferentes valores de severidade sendo nota 1: ausência de doença; nota 2: de 1\% a 15\% da área foliar afetada; nota 3: de 15\% a 18\% da área foliar afetada; nota 4: de 18\% a 30\% da área foliar afetada; nota 5: de 30\% a 50\% da área foliar afetada e nota 6: de 50\% a 60\% da área foliar afetada.

No segundo experimento, a cultivar Piatã IAC 387 teve a menor incidência da doença na primeira avaliação $(21,1 \%)$, e foi seguida por Ouro Verde IAC H5010-5 (46,7\%), Icatu Vermelho IAC $4045(48,9 \%)$, Icatu Precoce IAC $3282(50,0 \%)$ e Tupi IAC 1669-33 (55,5\%). Nas mudas dos materiais Robusta IAC1653-7, Mundo Novo IAC 388-17-1, Hibrido de Timor IAC 1559-13, Apoatã IAC 2258, Bourbon Amarelo, Mundo Novo IAC 376-4, Bourbon Vermelho, Catuaí Amarelo IAC 62 e Catuaí Vermelho IAC 144, houve maior incidência da doença nessa avaliação com 93,3; 88,9; 83,3; 76,7; 76,7; 68,9 e 65,6
\% respectivamente. A progênie Robusta IAC1653-7 teve a maior severidade do experimento $(3,64)$, sendo seguida pelos materiais Bourbon Amarelo $(2,99)$, Híbrido de Timor IAC 1559-13 $(2,97)$, Bourbon Vermelho $(2,91)$, Mundo Novo IAC 376-4 (2,86), Apoatã IAC $2258(2,79)$ e Mundo Novo IAC 388-17-1 (2,63). Os cafeeiros de Piatã IAC 387, Icatu Vermelho IAC 4045, Icatu Precoce IAC 3282, Ouro Verde IAC H5010-5 e Tupi IAC 166933 , tiveram os menores valores de severidade da doença (notas entre 1,28 e 1,88). As cultivares Catuaí Vermelho IAC 144 e Catuaí Amarelo IAC 62 tiveram valores 
Tabela 2. Incidência e severidade da cercosporiose em plantas de Coffea arabica, C. canephora e híbridos interespecíficos avaliadas no primeiro experimento, realizado de abril a maio de 2003

\begin{tabular}{|c|c|c|c|}
\hline Espécies e híbridos & Cultivares & Incidência $^{(1)}$ & Severidade ${ }^{(2)}$ \\
\hline \multirow[t]{11}{*}{ Coffea arabica } & Bourbon Amarelo & $63,1 \mathrm{a}^{3,4}$ & $2,92 \mathrm{a}^{4}$ \\
\hline & Bourbon Vermelho & $70,0 \mathrm{a}$ & $3,37 \mathrm{a}$ \\
\hline & Mundo Novo IAC 388-17-1 & $53,2 \mathrm{~b}$ & $2,34 \mathrm{c}$ \\
\hline & Mundo Novo IAC 376-4 & $63,8 \mathrm{~b}$ & $2,71 \mathrm{~b}$ \\
\hline & Catuaí Vermelho IAC 144 & $33,8 \mathrm{c}$ & $1,75 \mathrm{~d}$ \\
\hline & Catuaí Amarelo IAC 62 & $52,7 \mathrm{~b}$ & $2,29 \mathrm{c}$ \\
\hline & Icatu Precoce IAC 3282 & $63,9 \mathrm{~b}$ & $2,50 \mathrm{~b}$ \\
\hline & Icatu Vermelho IAC 4045 & $43,6 \mathrm{c}$ & $2,07 \mathrm{c}$ \\
\hline & Obatã IAC 1669-20 & $54,7 \mathrm{~b}$ & $2,27 \mathrm{c}$ \\
\hline & Ouro Verde IAC H5010-5 & $31,2 \mathrm{c}$ & $1,65 \mathrm{~d}$ \\
\hline & Tupi IAC 1669-33 & $28,4 \mathrm{c}$ & $1,47 \mathrm{~d}$ \\
\hline C. canephora & Apoatã IAC 2258 & $74,8 \mathrm{a}$ & $2,84 \mathrm{~b}$ \\
\hline C. canephora & Robusta IAC1653-7 & $81,6 \mathrm{a}$ & $3,42 \mathrm{a}$ \\
\hline C. arabica $\times$ C.canephora & Hibrido de Timor IAC 1559-13 & $65,5 \mathrm{~b}$ & $2,87 \mathrm{~b}$ \\
\hline C. arabica $x$ C. dewevrei & Piatã IAC 387 & $35,5 \mathrm{c}$ & $1,73 \mathrm{~d}$ \\
\hline Coeficiente de variação (\%) & & 41,4 & 34,9 \\
\hline
\end{tabular}

(1) Incidência - porcentagem de folhas com lesões por planta.

(2) Severidade - nota média $\left.=\mathrm{n}_{1} \times 1+\mathrm{n}_{2} \times 2+\mathrm{n}_{3} \times 3+\mathrm{n}_{4} \times 4,+\mathrm{n}_{5} \times 5+\mathrm{n}_{6} \times 6\right) / \mathrm{N}$, em que $\mathrm{n}_{1}, \mathrm{n}_{2}, \mathrm{n}_{3,}, \mathrm{n}_{4,} \mathrm{n}_{5}, \mathrm{n}_{6}=$ número de folhas da planta com as notas 1, 2, 3, 4, 5 e 6; e $\mathrm{N}=$ número total de folhas por amostra

$\left({ }^{3}\right)$ Análise com os dados transformados para $\sqrt{x}+1$.

$\left({ }^{4}\right)$ Médias comparadas pelo teste de Scott-Knott a 5\% de probabilidade.

Tabela 3. Incidência e severidade da cercosporiose em plantas de Coffea arabica, C. canephora e híbridos interespecíficos avaliadas no segundo experimento, realizado de julho a agosto de 2003

\begin{tabular}{|c|c|c|c|c|c|}
\hline \multirow[t]{2}{*}{ Espécies e híbridos } & \multirow[t]{2}{*}{ Tratamentos } & \multicolumn{2}{|c|}{$\begin{array}{c}\text { Primeira avaliação } \\
\text { (10 dias após inoculação) }\end{array}$} & \multicolumn{2}{|c|}{$\begin{array}{c}\text { Segunda avaliação } \\
\text { (16 dias após inoculação) }\end{array}$} \\
\hline & & Incidência $^{(1)}$ & Severidade ${ }^{(2)}$ & Incidência ${ }^{(1)}$ & Severidade (2) \\
\hline \multirow[t]{11}{*}{ Coffea arabica } & Bourbon Amarelo & $83,3 a^{3,4}$ & $2,99 b^{4}$ & $92,2 a^{3,4}$ & $4,73 \mathrm{a}^{4}$ \\
\hline & Bourbon Vermelho & 76,7 a & $2,91 \mathrm{~b}$ & $95,6 \mathrm{a}$ & $5,07 \mathrm{a}$ \\
\hline & Mundo Novo IAC 388-17-1 & 88,9 a & $2,63 \mathrm{~b}$ & $96,7 \mathrm{a}$ & $4,32 \mathrm{a}$ \\
\hline & Mundo Novo IAC 376-4 & $76,7 \mathrm{a}$ & $2,86 \mathrm{~b}$ & 88,9 a & $4,39 \mathrm{a}$ \\
\hline & Catuaí Vermelho IAC 144 & $65,6 \mathrm{a}$ & $2,37 \mathrm{c}$ & 83,3 a & $3,58 \mathrm{~b}$ \\
\hline & Catuaí Amarelo IAC 62 & $68,9 \mathrm{a}$ & $2,24 \mathrm{c}$ & 85,6 a & $3,64 \mathrm{~b}$ \\
\hline & Icatu Precoce IAC 3282 & $50,0 \mathrm{~b}$ & $1,76 \mathrm{~d}$ & $68,9 \mathrm{~b}$ & $2,79 \mathrm{c}$ \\
\hline & Icatu Vermelho IAC 4045 & $48,9 \mathrm{~b}$ & $1,71 \mathrm{~d}$ & $74,4 \mathrm{~b}$ & $3,06 \mathrm{c}$ \\
\hline & Obatã IAC 1669-20 & $66,7 \mathrm{a}$ & $2,29 \mathrm{c}$ & 93,3 a & $4,23 \mathrm{a}$ \\
\hline & Ouro Verde IAC H5010-5 & $46,7 \mathrm{~b}$ & $1,77 \mathrm{~d}$ & $76,7 \mathrm{~b}$ & $3,01 \mathrm{c}$ \\
\hline & Tupi IAC 1669-33 & $55,6 \mathrm{~b}$ & $1,88 \mathrm{~d}$ & $65,6 \mathrm{~b}$ & $2,61 \mathrm{c}$ \\
\hline \multirow[t]{2}{*}{ C. canephora } & Apoatã IAC 2258 & 83,3 a & $2,79 \mathrm{~b}$ & 93,3 a & $3,72 \mathrm{~b}$ \\
\hline & Robusta IAC1653-7 & $93,3 \mathrm{a}$ & $3,64 \mathrm{a}$ & 97,8 a & $4,78 \mathrm{a}$ \\
\hline C. arabica $\mathrm{x}$ C. canephora & Hibrido de Timor IAC 1559-13 & 85,6 a & $2,97 \mathrm{~b}$ & $96,7 \mathrm{a}$ & 4,69 a \\
\hline C. arabica $\times$ C. dewevrei & Piatã IAC 387 & $21,1 \mathrm{c}$ & $1,28 \mathrm{~d}$ & $48,9 \mathrm{c}$ & $2,02 \mathrm{c}$ \\
\hline Coeficiente de variação (\%) & & 43,2 & 36,8 & 27,9 & 32,1 \\
\hline
\end{tabular}

(1) Incidência - porcentagem de folhas com lesões por planta.

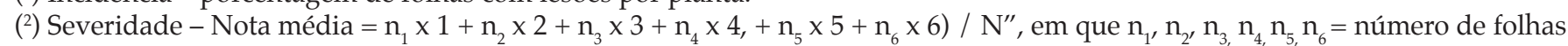
da planta com as notas 1, 2, 3, 4, 5 e 6 ; e $\mathrm{N}$ = número total de folhas por amostra.

$\left({ }^{3}\right)$ Análise com os dados transformados para $\sqrt{x}+1$.

$\left({ }^{4}\right)$ Médias comparadas pelo teste de Scott-Knott a 5\% de probabilidade. 
intermediários de severidade, com notas de 2,24 e 2,37 respectivamente, embora estivessem entre as cultivares com as mais elevadas incidências da doença $(65,6 \%$ e $68,9 \%$ ) (Tabela 3). Na segunda avaliação, a progênie Robusta IAC1653-7, as cultivares Mundo Novo IAC 388-17-1, Mundo Novo IAC 376-4, Bourbon Amarelo, Bourbon Vermelho, Obatã IAC 1669-20 e o Híbrido de Timor IAC 1559-13 mantiveram os mais elevados valores de incidência e severidade da cercosporiose, que variaram entre 97,8 e $83,3 \%$ de incidência e entre 5,07 e 4,32 de severidade. Nas cultivares Apoatã IAC 2258, Catuaí Amarelo IAC 62 e Catuaí Vermelho IAC 144, notou-se elevada incidência (93,3; 85,6 e 83,3 respectivamente), porém com níveis de severidade intermediários $(3,72 ; 3,64$ e 3,58 respectivamente). O café Piatã IAC 387 manteve a menor incidência da doença neste experimento $(48,9 \%)$ e este híbrido, acrescido das cultivares Tupi IAC 1669-33, Icatu precoce IAC 3282, Ouro Verde IAC H5010-5 e Icatu Vermelho IAC 4045, possuem os menores valores de severidade com notas entre 2,02 e 3,06 (Tabela 3). Nesse experimento, foram observados elevados valores de incidência e severidade da cercosporiose, provavelmente porque o potencial de inóculo foi elevado, as condições ambientais em que as plantas permaneceram foram favoráveis à doença e porque as folhas inoculadas foram apenas as mais jovens de cada muda, as quais são mais suscetíveis. Em estudo de RENFIFO-GuZMAn et al., (2006) foi verificado que as folhas mais jovens de mudas de cafeeiros possuem níveis mais elevados de severidade e menor período de incubação da cercosporiose.

As seleções de C. canephora avaliadas neste estudo, Robusta IAC 1653-7 e Apoatã IAC 2258, foram muito suscetíveis à cercosporiose. A primeira cultivar revelou os maiores valores de incidência $(81,6 \%$ e $93,3 \%)$ e severidade $(3,42$ e 3,64$)$ da doença, nos dois experimentos realizados. Os representantes da espécie C. canephora são fontes de resistência genética para nematóides dos gêneros Meloydogine exigua, M. incognita, M. paranaensis e Pratylenchus coffeae (Medina Filho et al., 1995; FAzUOLI et al., 2004) e ferrugem (FAzUOLI et al., 2004; FAzUOLI et al.2005), contribuindo sobremaneira para os programas de melhoramento do cafeeiro (FAZUOLI et al., 2005). Assim, sugere-se o estudo de outros representantes desta espécie visando identificar possíveis fontes de resistência à cercosporiose, considerando-se sua importância para o melhoramento do cafeeiro e a grande variabilidade que pode proporcionar especialmente por se tratar de uma espécie alógama.

Nas cultivares Bourbon Vermelho e Bourbon Amarelo constatou-se moderada a elevada suscetibilidade à cercosporiose neste estudo (entre 83,3\% e 63,1\% de incidência da doença). Embora estas cultivares sejam também muito suscetíveis à ferrugem
(GUERREIRO FilHo et al., 2006), são ainda extensamente cultivadas no parque cafeeiro brasileiro, especialmente por serem consideradas como excelentes em relação à qualidade da bebida (FAzUOLI et al., 2004). Como a produção de cafés de qualidade tem crescido no Brasil, sugere-se que seja feita, sempre que possível, a avaliação da cercosporiose nos programas de melhoramento dessas cultivares.

O Híbrido de Timor IAC 1559-13 revelou-se como suscetível à cercosporiose (incidência entre $85,6 \%$ e $65,5 \%$ ). Esse material, provavelmente, um híbrido natural de C. canephora e C. arabica, que possui 44 cromossomos, é autofértil (Silvarolla et al., 1997) e relevante fonte de resistência nos programas de melhoramento a $H$. vastarix, $M$. exigua e Colletotrichum kahawae (BetTencourt e CARVAlHo, 1968). É importante assinalar que são utilizadas diferentes seleções do Híbrido de Timor nos programas de melhoramento do café no Brasil e que seu comportamento e de suas progênies com relação à cercosporiose pode ser variável. Por exemplo, Araújo NetTo et al. (1978) observaram, em condições de campo, que duas introduções do Híbrido de Timor possuíam diferentes níveis de resistência à cercosporiose. O Híbrido de Timor UFV 382 foi mais suscetível à doença do que o Híbrido de Timor UFV 409, com incidência de $32,4 \%$ e 21,6\%, respectivamente, eesses materiais, assim como o Híbrido de Timor do presente estudo, foram mais suscetíveis que as cultivares Catuaí Vermelho, Catuaí Amarelo e uma seleção de Icatu, que tiveram 13,6; 14,9 e 18,5\% de incidência respectivamente. FERNANDES et al. (1990) verificaram diferentes níveis de resistência à cercosporiose em progênies de Catimor oriundas de cruzamentos entre Caturra Vermelho ou Amarelo e Híbrido de Timor. Algumas progênies possuíam nível de resistência semelhante ao da cultivar Catuaí Vermelho IAC 144. Como essas progênies exibem grande variabilidade e a cercosporiose é importante para a cultura do cafeeiro, sugere-se que, sempre que possível, a reação a esta doença seja observada nas progênies derivadas do Híbrido de Timor.

Entre as cultivares mais presentes no parque cafeeiro brasileiro, Mundo Novo IAC 388-17-1 e Mundo Novo IAC 376-4 revelaram-se como suscetíveis à cercosporiose (entre $53,2 \%$ e $88,9 \%$ de incidência), e as cultivares Catuaí Vermelho IAC 144 e Catuaí Amarelo IAC 62, como moderadamentesuscetíveisà cercosporiose (entre $33,8 \%$ e $68,9 \%$ de incidência). As duas últimas tiveram menores valores de severidade (entre 1,75 e 2,37 ) do que as cultivares do grupo Mundo Novo (entre 2,34 e 2,86). Essa reação à doença era esperada, pois em estudo anterior realizado por ARAúJo NeTTO et al. (1976), as cultivares Catuaí Vermelho e Catuaí Amarelo foram as menos afetadas pela cercosporiose, com incidências de $13,6 \%$ e $15 \%$ respectivamente. Em experimento 
realizado com mudas de cafeeiro, FERNANDEs et al., (1990) verificaram na cultivar Catuaí IAC 144 o menor índice de severidade da cercosporiose.

As cultivares Icatu Precoce IAC 3282 e Icatu Vermelho IAC 4045 foram moderadamente suscetíveis à cercosporiose (entre 50,0\% e 48,9\% de incidência). No primeiro experimento, a cultivar Icatu Vermelho IAC 4045 teve menor incidência $(43,6 \%)$ e severidade $(2,07)$ do que a cultivar Icatu Precoce IAC 3282 (63,9\% de incidência e 2,5 de severidade). Em estudo anterior, ARAúo NetTo et al. (1976) observaram diferente comportamento em relação à cercosporiose entre outras cultivares do grupo Icatu, desde alta suscetibilidade (38,2\% de incidência) a resistência moderada (15,6\% de incidência). As cultivares Icatu Precoce IAC 3282 e Icatu Vermelho IAC 4045 foram menos afetadas pela cercosporiose que as cultivares do grupo Catuaí e Mundo Novo, no segundo experimento. O mesmo ocorreu no estudo de Pozza et al. (2004), no qual a cultivar Icatu foi mais resistente à cercosporiose que as cultivares Mundo Novo e Catuaí. Como inserir as cultivares do grupo Icatu são originárias de cruzamentos entre $C$. canephora e $C$. arabica, a variabilidade na sua reação a doenças pode ocorrer e poderia ser explorada em estudos futuros, especialmente considerando que estas cultivares são consideradas moderadamente resistentes à ferrugem (FAZUOLI, 2004).

A cultivar Tupi IAC 1669-33 comportou-se como moderadamente resistente à cercosporiose $(28,4 \%$ a $55,5 \%$ de incidência) e a cultivar Obatã IAC 1669-20 como moderadamente suscetível $(54,7 \%$ a $66,7 \%$ de incidência). Essas cultivares foram originadas a partir de cruzamentos entre $C$. arabica e o Híbrido de Timor CIFC832/ 2 sendo resistentes à ferrugem (FAZUOLI, 2004). Diferenças nos níveis de suscetibilidade à cercosporiose nas progênies originadas desse híbrido são esperadas, especialmente porque se trata de material com elevada variabilidade natural. Também ressalta-se que o Híbrido de Timor CIFC 832/2 utilizado nos cruzamentos para a obtenção dessas cultivares, é diferente do Híbrido de Timor CIFC 1559-13, que no presente estudo foi muito suscetível à doença. Fernandes et al. (1990) já havia verificado diferentes reações à cercosporiose em progênies de cruzamentos entre Híbrido de Timor e a cultivar Caturra.

Neste estudo, a cultivar Tupi IAC 1669-33 teve, relativamente, baixos níveis de incidência e severidade da cercosporiose $(28,4 \%$ a $55,5 \%$ de incidência). Por esta razão, essa cultivar pode ser promissora para lavouras ou regiões muito propícias à cercosporiose e para cultivos orgânicos de cafeeiro, nos quais a ferrugem e a cercosporiose podem ser fatores limitantes.

A cultivar Ouro Verde H5010-5, um cruzamento de Catuaí Amarelo e Mundo Novo IAC 515 (FAzuoli,
2004), embora seja suscetível à ferrugem, esteve entre as cultivares com menor incidência e severidade da cercosporiose (31,2\% a 46,7\% de incidência; 1,65 a 1,77 de severidade). É interessante, em estudos futuros, que esta cultivar, assim como Tupi IAC 1669-33, sejam avaliadas em condições de campo para verificar se mantêm a tendência de alguma resistência à cercosporiose.

O café Piatã IAC 387, um híbrido natural de $C$. dewevrei e C. arabica, teve a menor incidência e severidade da cercosporiose nos experimentos realizados $(35,5 \%$ a 21,1\% de incidência; 1,73 e 1,28 de severidade). Este material, utilizado como fonte de resistência a $M$. exigua (FAZUOLI, 1999), após avaliação em condições de campo, também poderá ser empregado como fonte de resistência à cercosporiose em programas de melhoramento do cafeeiro ou ainda como padrão de resistência à doença em estudos futuros.

Os resultados deste estudo precisam ser interpretados com cuidado, pois se trata de experimentos realizados com mudas e o comportamento das cultivares pode variar quando cultivados no campo ou mesmo de região para região. Entretanto, dificilmente se conseguiriam lavouras com todos estes materiais com a mesma idade e, em condições de campo, outros fatores como a nutrição dos cafeeiros poderiam interferir nos resultados. Neste estudo, tomou-se o cuidado em produzir as mudas simultaneamente e em substratos homogêneos, sendo mantidas em casa de vegetação sem a aplicação de fungicidas e/ou inseticidas que pudessem interferir nos resultado, até durante a realização dos experimentos. Por estas razões, pode-se considerar que este trabalho representa uma primeira avaliação com relação à resistência à cercosporiose de cultivares de cafeeiro extensamente cultivadas no Brasil, além de espécies de Coffea relevantes para os programas de melhoramento do IAC. Adicionalmente, o desenvolvimento dos materiais foi semelhante nos dois experimentos realizados, o que aumenta a possibilidade de que os resultados deste estudo sejam reproduzidos em condições de campo.

\section{CONCLUSÕES}

1. O café Piatã IAC 387 e as cultivares Ouro Verde e Tupi IAC 1669-33 são parcialmente resistentes à cercosporiose.

2. As progênies de C. canephora, Robusta IAC 1653-7 e Apoatã IAC 2258, seguidas pelo Híbrido de Timor IAC 1559-13 e pelas cultivares de C. arabica, Bourbon Amarelo e Bourbon Vermelho são bastante suscetíveis à cercosporiose.

3. As cultivares Catuaí Vermelho IAC 144 e Catuaí Amarelo IAC 62 possuem maiores níveis de resistência 
à cercosporiose do que as cultivares Mundo Novo IAC 388-17-1 e Mundo Novo IAC 376-4.

\section{REFERÊNCIAS}

AGUIAR, A.T.E.; GUERREIRO FILHO, O.; MALUF, M.P.; GALLO, P.B.; FAZUOLI, L.C. Caracterização de cultivares de Coffea arabica mediante a utilização de descritores mínimos. Bragantia, v.63, p.179-192, 2004.

ARAÚJO NETTO, K.; MIGUEL, A.E.; FERREIRA, A.J. Comportamento de progênies de Catimor, Icatu, Catuaí e Mundo Novo e outras em relação à cercosporiose. In: CONGRESSO BRASILEIRO DE PESQUISAS CAFEEIRAS, 6., 1978. Ribeirão Preto. Resumos... Rio de janeiro, IBC-GERCA, 1978. p.278-279.

BETTENCOURT, A.J.; CARVALHO, A. Melhoramento visando a resistência do cafeeiro à ferrugem. Bragantia, v.27, p.35-68, 1968.

CARVALHO, V.L.; CUNHA R.L.; CHALFOUN S.M. Manejo ecológico das principais doenças do cafeeiro. Informe Agropecuário, v. 23, p.101-114, 2002.

DHINGRA, O.D.; SINCLAIR J.B. Basic plant pathology methods. Second edition. CRC Lewis Publishers: Boca Raton, 1995. 434p.

FAZUOLI, L.C. Cultivares de café IAC. O Agronômico, v.51, p.5-7, 1999.

FAZUOLI, L.C. Melhoramento genético do cafeeiro. In: RIFIB - Reunião Anual do Instituto Biológico, v.10, p.1-15, 2004.

FAZUOLI, L.C.; MEDINA FILHO, H.P.; GUEREIRO FILHO, O.; GONÇALVES, W.; SILVAROLLA, M.B. Cultivares de Café. Disponível em: http://www.iac.sp.gov.br/centros/ centro_cafe/Caf\%C3\%A9.htm> Acesso em: 2/5/2008.

FAZUOLI, L.C.; OLIVEIRA, A.C.B.; BRAGHINI, M.T.; SILVAROLLA, M.B. Identification and use of sources of durable resistance to coffee leaf rust at the IAC. In: ZAMBOLIM, L.; ZAMBOLIM, E.M.; VÁRZEA, V.M. Durable resistance to coffee leaf rust. Viçosa: Universidade Federal de Viçosa, Departamento de Fitopatologia, 2005. p.137-185.
FERNANDES, A.T.F.; VALE, F.X.R.; PELOSO, M.C.; ZAMBOLIM, L.; MAFFIA, L.A.; PEREIRA, A.A.; CHAVES, G.M.; CRUZ FILHO, J. Resistência de progênies de Catimor a diferentes isolados de Cercospora coffeicola Berk. \& Look. Fitopatologia Brasileira, v. 15, p.45-49, 1990.

GUERREIRO FILHO, O; FAZUOLI, L.C.; AGUIAR, A.T.E. Cultivares de Coffea arabica selecionadas pelo IAC: características botânicas, tecnológicas, agronômicas e descritores mínimos. http://www.infobibos.com/artigos/2006_2/ cultivares_cafe/ index.htm $>$ Acesso em: 2/5/2008.

MEDINA FILHO, H.P.; CARVALHO, A.; BALLVE, R.M.L.; BORDIGNON, R.; SILVAROLLA, M.B.; LIMA, M.M.A.; FAZUOLI, L.C. Evidência isoenzimática sobre a origem interespecífica do café Piatã. Bragantia, v.54, p.263-273, 1995.

MORAES, S.A. Quantificação de doenças de plantas. 2007. Artigo em Hypertexto. Disponível em: http://www. infobibos.com/Artigos/2007_1/doencas / index.htm> Acesso em: $12 / 12 / 2008$.

POZZA, A.A.A.; AVES, E.; POZZA, E.A.; CARVALHO, J.G.; MONTANARI, M.; GUIMARÃES, P.T.G.;SANTOS, D.M. Efeito do silício no controle da cercosporiose em três variedades de cafeeiro. Fitopatologia Brasileira, v.29, p.185-188, 2004.

RENGIFO-GUZMAN, H.G.; LEGUIZAMÓN-CAYEDO, J.E.; RIAÑO-HERRERA, N.M. Incidencia e severidad de La mancha de hierro em plântulas de Coffea arabica em diferentes condiciones de nutrición. Cenicafe, v.57, p.232-242, 2006.

SILVAROLLA, M.B.; GUERREIRO FILHO, O.; LIMA, M.A.L.; FAZUOLI, L.C. Avaliação de progênies derivadas do híbrido timor com resistência ao agente da ferrugem. Bragantia, v.56, p.47-58, 1997.

THOMAZIELLO, R.A., FAZUOLI, L.C., PEZZOPANE, J.R., FAHL, J.I., CARELLI, M.L.C. Café arabica: cultura e técnicas de produção. Campinas: 2000. Instituto Agronômico, 82 p. (Boletim Técnico n. ${ }^{\circ}$ 187)

ZAMBOLIM L.; VALE F.X.R.; ZAMBOLIM E.M. Doenças do cafeeiro. In: KIMATI, H.; AMORIM, L.; REZENDE, J.A.M.; BERGAMIN FILHO, A.; CAMARGO, L.E. (Ed.). Manual de Fitopatologia. vol. 2: Doenças das plantas cultivadas. 4.ed. São Paulo: Ceres. 2005. p.165-180. 